\title{
Self-sensing dielectric elastomer actuators in closed-loop operation
}

Rosset, Samuel; O’Brien, Benjamin M; Gisby, Todd; Xu, Daniel; Shea, Herbert R.; Anderson, Iain A.

- Published in: Smart Materials and Structures (ISSN: 1361-665X), vol. 22, num. 10, p. 104018

- Bristol: Iop Publishing Ltd, 2013

Because of their large output strain, dielectric elastomer actuators (DEAs) have been proposed for tunable optics applications such as tunable gratings. However, the inherent viscoelastic drift of these actuators is an important drawback and closed-loop operation of DEAs is a prerequisite for any accurate real-world application. In this paper, we show how capacitive self-sensing can be used to drive a DEA in closed-loop without the need for any external sensor. The method has been demonstrated on a DEA tunable grating based on a VHB acrylic and silicone membrane. The results show that the widely used VHB presents a time-dependent drift between the capacitance of the electrodes and their strain. The silicone-based grating does not exhibit such a drift, and its strain can be stabilized by regulating the capacitance of the device to a constant value. We also report on an new fabrication method for thin deformable gratings based on replication on a water-soluble master and a $27 \%$ change in the grating period has been obtained on a VHB-based device.

\section{Reference}

- $\quad$ doi:10.1088/0964-1726/22/10/104018

(C) Iop Publishing Ltd, 2013 


\title{
Self-sensing dielectric elastomer actuators in closed loop operation
}

\author{
Samuel Rosset ${ }^{1}$, Benjamin M. O’Brien ${ }^{2}$, Todd Gisby ${ }^{2}$, Daniel $\mathrm{Xu}^{2}$, \\ Herbert R. Shea ${ }^{1}$ and Iain A. Anderson ${ }^{2}$ \\ ${ }^{1}$ Ecole polytechnique fédérale de Lausanne (EPFL) Jaquet-Droz 1, 2002 Neuchâtel, Switzerland \\ ${ }^{2}$ University of Auckland, Biomimetics Laboratory, Auckland Bioengineering Institute, 70 \\ Symonds Street, Auckland, New-Zealand \\ E-mail: samuel.rosset@a3.epfl.ch
}

\begin{abstract}
Because of their large output strain, dielectric elastomer actuators (DEAs) have been proposed for tunable optics applications such as tunable gratings. However, the inherent viscoelastic drift of these actuators is an important drawback and closed-loop operation of DEAs is a prerequisite for any accurate real-world application. In this paper, we show how capacitive self-sensing can be used to drive a DEA in closed-loop without the need for any external sensor. The method has been demonstrated on a DEA tunable grating based on a VHB acrylic and silicone membrane. The results show that the widely used VHB presents a time-dependent drift between the capacitance of the electrodes and their strain. The silicone-based grating does not exhibit such a drift, and its strain can be stabilized by regulating the capacitance of the device to a constant value. We also report on an new fabrication method for thin deformable gratings based on replication on a water-soluble master and $27 \%$ change in the grating period has been obtained on a VHB-based device.
\end{abstract}

PACS numbers: 85.50.-n,77.84.Jd

\section{Introduction}

Dielectric elastomer actuators (DEAs) are soft transducers capable of important strains typically larger than 10\%, but which can exceed $1000 \%$ depending on the configuration [1]. A DEA consists of a rubbery capacitor made from a soft dielectric elastomer membrane sandwiched between two compliant electrodes [2,3]. When a voltage is applied between the electrodes, the generated electrostatic stress compresses the dielectric membrane, which reduces in thickness and expands in area. The large achievable strain of these actuators, combined with their soft rubbery nature and low weight has prompted the development of a broad range of applications such as energy harvesters [4-6], biological cell stretchers [7], haptic feedback devices [8,9], rotary motors [10], and other soft and smart machines [11].

One field that is of particular interest for DEAs is tunable optics, and several soft tunable devices have been developed, taking advantage of the large tuning range, softness and intrinsic transparency of the elastomeric layers: tunable lenses [12], tunable gratings [13-15], and tunable phase retarders [16] have already been reported in the literature. 


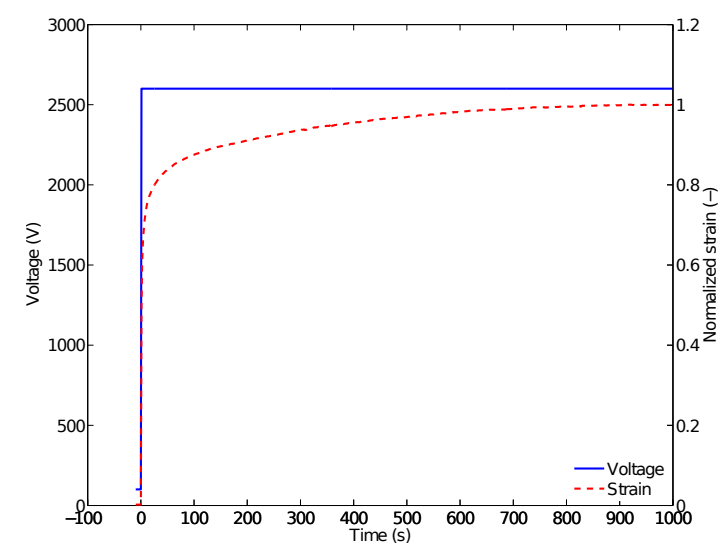

Figure 1. Illustration of the viscoelastic response of a VHB-based DEA when submitted to a voltage step. The strain increases rapidly during the first few seconds, but then continues to slowly increase during the observation period ( $15 \mathrm{~min}$ ). It takes 163 seconds for the actuator to reach $90 \%$ of the final displacement.

However, DEAs suffer from an important drawback detrimental to many applications requiring a precise positioning: the viscoelastic behaviour of the elastomeric membranes, which leads to a time-dependent displacement. Three classes of material are mainly used as dielectric membrane for DEAs: silicones, polyurethanes, and the proprietary acrylic elastomer Very High Bond (VHB) adhesive tape from $3 \mathrm{M}[17,18]$. The latter being the most widely used in the laboratory, mainly due to its outstanding output strain, its intrinsic adhesive nature which facilitates rapid prototyping, and because it is readily available in membrane form, whereas silicones and polyurethanes must be processed into thin-films, which requires time and equipment. Unfortunately, VHB is highly viscoelastic, and therefore presents a time-dependent strain when submitted to a stress step input: VHB-based actuators react slowly and do not keep a stable position when driven with a constant voltage. This behaviour is illustrated on figure 1, which shows the normalized strain response of a DEA made with a VHB 4905 membrane (500 $\mu \mathrm{m}$ thick), prestretched to 3 times its initial size in both $\mathrm{x}$ and $\mathrm{y}$ direction (final thickness: $55.6 \mu \mathrm{m}$ ) and coated with Nyogel 756 grease electrodes.

The optical applications mentioned above (tunable lens, grating and phase retarder) can exhibit a very large tuning range when based on dielectric elastomer actuation, but they also require to be able to hold a precise position without drift, which a DEA cannot do. This can naturally be generalized outside the sphere of tunable optical devices to encompass the complete field of DEAs: in addition to the large strain that this family of actuators can provide, it is often important to be able to precisely control the output strain and to keep it stable in time. This important issue, which remains largely ignored in the DEA-related literature, prevents any realistic use of this technology to build precise products.

\subsection{Closed-loop operation}

One possible solution to improve the stability and precision of DEAs is to use them in closedloop operation. This is usually done by measuring the parameter of interest (for example the displacement of an actuator), calculating the error with a desired set point, and applying an input voltage which reduces the error signal so that it approaches zero. Closed-loop control has already 


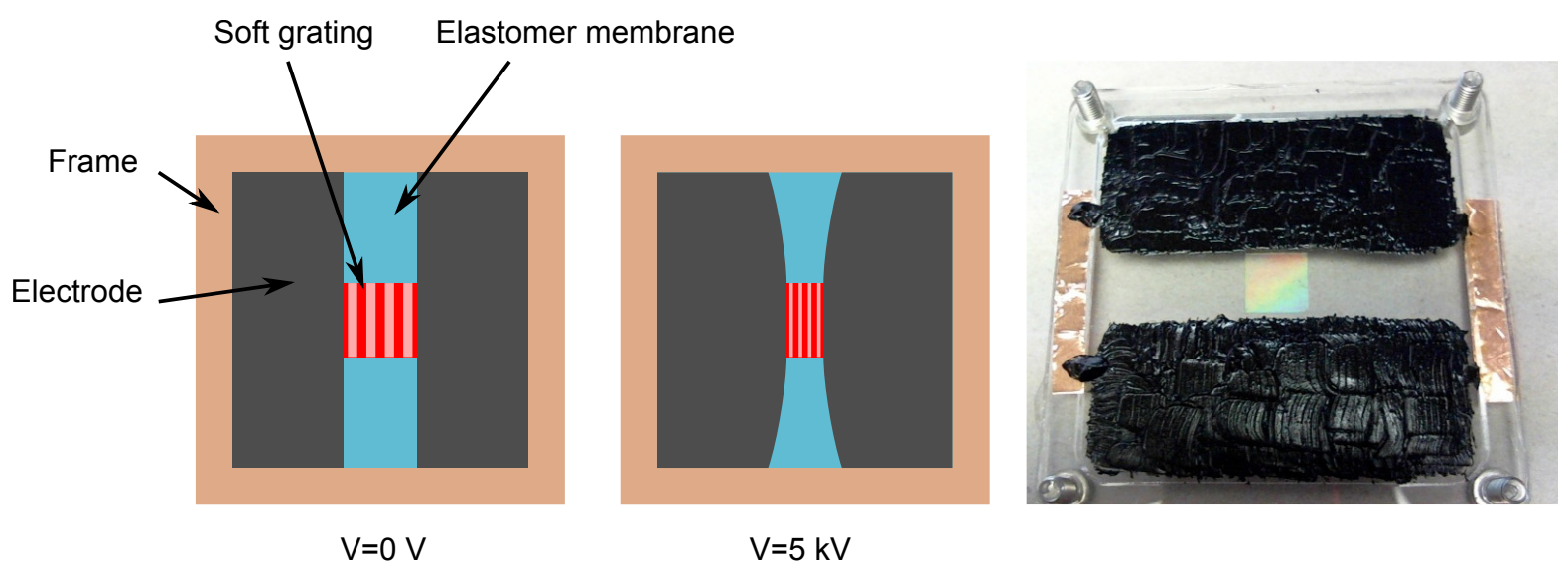

Figure 2. DEA-based tunable grating: An elastomer membrane is stretched on a frame. A soft grating is replicated into an elastomer and placed at the center of the membrane. Two pairs of electrodes are patterned, on each side of the grating, parallel to the grating structure. When a voltage is applied to the electrodes, the grating is compressed and its period changes. Right: picture of the fabricated device

a)

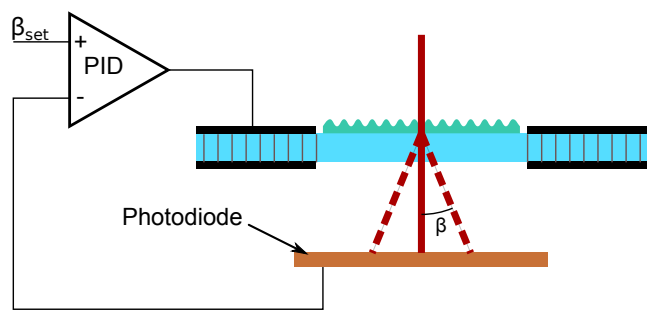

b)

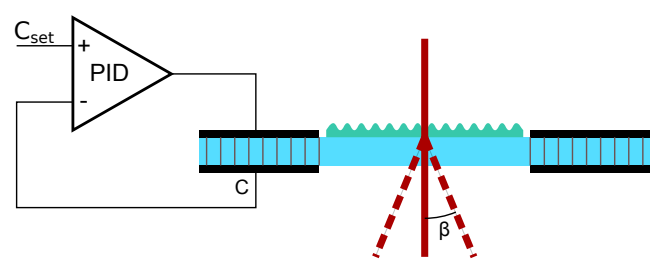

Figure 3. Illustration of a DEA tunable grating used in closed-loop mode. a) classical approach using an external sensor to measure the first order diffraction angle, comparing it to the desired setpoint, and setting the driving voltage accordingly. b) closed-loop operation via self-sensing of the electrode capacitance. No external sensor is required for this approach.

been applied to DEAs to improve their stability, using external sensors to measure the strain and/or the force [19-22] The most common type of controller for closed loop operation is the PID (proportional-integral-derivative) controller, which uses the instantaneous value of the error, as well as its time-history and rate of change to compute the driving signal, in order to reach the setpoint value rapidly, with limited overshoot and without steady-state error.

To investigate the use of closed-loop operation for precise control of DEA, we have developed a tunable grating, which is a typical example of an application that can greatly benefit from large tuning range, but which also requires a precise control of the actuation strain over time. The device architecture is similar to the work of Kollosche et al. [15] and consists of a membrane prestretched on a frame with electrodes on the side and a soft, deformable grating at the center (figure 2).

In the case of the tunable grating (figure 2), the classical approach for operating it in closedloop mode consists in adding an external sensor, such as a photo-diode to measure the first order diffraction angle (figure $3 \mathrm{a}$ ). This value is fed to the controller, which evaluates the error with respect to the set point and calculates the appropriate driving voltage. However, this approach involves the use of an external sensor to measure a process variable (the diffraction angle in this 


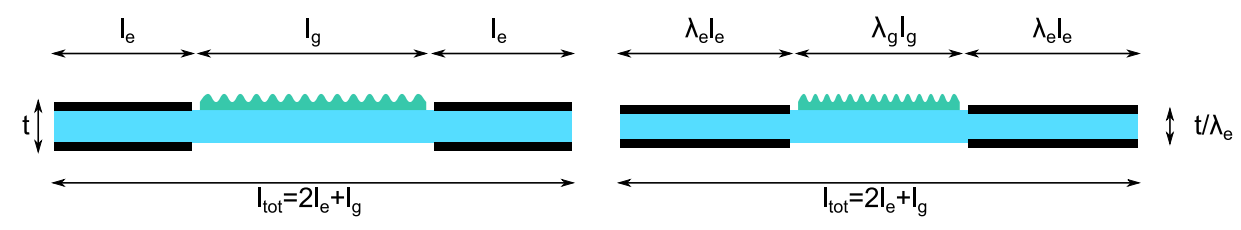

Figure 4. Geometric relation between the strain of the electrodes and the strain of the central grating. The left figure represents the rest position at $0 \mathrm{~V}$, and the right figure shows the deformed configuration for $V>0$.

case), which adds complexity to the system and increases the cost. This in turns contradicts the main argument for a low-cost polymer based approach.

Another possibility is to use the actuator itself as a smart device, not only able to move, but to sense its own strain, through what is commonly known as self-sensing. The device plays the double role of actuator and integrated sensor, which makes the use of an external sensor unnecessary for closed-loop operation (figure $3 \mathrm{~b}$ ). The application of a voltage causes the electrodes of a DEA to expand in area and the dielectric membrane to decrease in thickness. This leads to a change of the electrical resistance of the electrodes, as well as a change of capacitance of the device, both of which can be used as a measure of the deformation of the device. Resistive self-sensing is not trivial to use, as it depends on many factors, such as strain, thickness of the electrodes (which can be difficult to control), resistivity of the electrode material (which can degrade with time or the number of cycles) and type of material used for the electrodes [23]. Capacitive sensing is easier to use, as the capacitance only depends on the geometry of the device: dielectric thickness and electrode surface, but not on their resistance or resistivity [24-26]. The relative permittivity of the dielectric may be unknown, but is assumed to be constant and not a function of the device deformation. Consequently, capacitive self-sensing has been chosen in the frame of this work.

For the capacitance to work as a proxy for the diffraction angle of the tunable grating, it is necessary to show that the two are directly linked, i.e. $\alpha=f(C)$, where $\alpha$ is the diffraction angle and $C$ the capacitance. Figure 4 shows the geometric relation between the stretch of the grating and the stretch of the electrodes. When the device is not activated, its total length $l_{\text {tot }}$ is given by the sum of the two electrodes $l_{e}$ and the grating $l_{g}$ :

$$
l_{\text {tot }}=2 l_{e}+l_{g}
$$

When a voltage is applied to the electrodes, they expend in the $x$ direction by a stretch $\lambda_{e}$, and the grating compresses by a stretch $\lambda_{g}<1$. Taking into account that the total length of the device doesn't change, the grating stretch can be expressed as:

$$
\lambda_{g}=2\left(1-\lambda_{e}\right) \frac{l_{e}}{l_{g}}+1
$$

As the electrodes extend from one side of the frame to the other in the $y$ (width) direction (see figure 2), the stretch in that direction is 1 . Elastomers have a very high bulk modulus, meaning that their volume remain constant when they are deformed. In the actuator zone of the membrane, the thickness stretch $\lambda_{z}$ induced by the deformation is therefore linked to the lateral stretch of the electrodes by the following relation:

$$
\lambda_{x} \lambda_{y} \lambda_{z}=1=\lambda_{e} \cdot 1 \cdot \lambda_{z} \Rightarrow \lambda_{z}=\lambda_{e}^{-1}
$$


Taking into account the relation between the lateral stretch and the thickness stretch, the capacitance $C$ of the device when it is activated is given by:

$$
C=\frac{\epsilon_{r} \epsilon_{0} y \lambda_{e} l_{e}}{t / \lambda_{e}}=\frac{\epsilon_{r} \epsilon_{0} y \lambda_{e}^{2} l_{e}}{t}=C_{0} \lambda_{e}^{2},
$$

where $\epsilon_{r}$ and $\epsilon_{0}$ are respectively the relative and vacuum permittivity, $t$ the initial thickness of the membrane without applied voltage, and $C_{0}$ a constant representing the initial capacitance of the device, when no voltage is applied. The first order diffraction angle $\alpha_{1}$ of the grating is defined by:

$$
\begin{aligned}
\alpha_{1} & =\arcsin \left(\frac{\tilde{\lambda}}{d}\right) \\
& =\arcsin \left(\frac{\tilde{\lambda}}{d_{0} \lambda_{g}}\right) \\
& =\arcsin \left(\frac{\tilde{\lambda}}{d_{0}\left(\left(1-\lambda_{e}\right) \frac{2 l_{e}}{l_{g}}+1\right)}\right) \\
& =\arcsin \left(\frac{\tilde{\lambda}}{d_{0}\left(\left(1-\sqrt{\frac{C}{C_{0}}}\right) \frac{2 l_{e}}{l_{g}}+1\right)}\right)
\end{aligned}
$$

where $\tilde{\lambda}$ is the wavelength of the light source, $d$ the period of the deformed grating, and $d_{0}$ the initial period of the grating, when no voltage is applied to the device. It can be seen that the first order diffraction angle $\alpha_{1}$ is expected to be only dependent on the capacitance of the device, as all the other parameters can - as a very good approximation - be considered as constants. Consequently, if the $\alpha_{1}=f(C)$ profile is known, the capacitance can then be used as a process variable for the controller. Instead of using a diffraction angle as a set point, a capacitance value is used.

An electronic self-sensing unit (SSU) $\ddagger$ capable of driving a DEA up to a voltage of $5 \mathrm{kV}$, while measuring characteristic parameters such as the capacitance, has been used in this investigation (figure 5). A real DEA device can be represented electrically by a capacitor $C$ in parallel with a resistor $R_{p}$ representing the leakage current through the dielectric. These two elements are connected in series with a second resistance $R_{s}$ representing the resistance of the electrodes [25]. These three elements have values which are dependent on the strain applied to the device. When the device is activated, the SSU adds a small time-varying signal to the main DC voltage signal used to drive the actuator. In addition, the unit measures the current flowing through the actuator. Using the voltage and current data, an algorithm based on multidimensional linear regression can calculate the value of the three electrical parameters in real time [27]. In the present beta stage of the unit, only the capacitance $C$ is calibrated, and it can be measured with a resolution of $1 \mathrm{pF}$ at a $100 \mathrm{~Hz}$ data acquisition rate. One notable advantage of the method is that it does not require any additional wire to the device for the sensing: only the two wires used to drive the actuator are necessary.

The SSU has an USB port which allows it to exchange information with a PC. A Labview interface was written to implement the controller feedback loop, reading the device capacitance from the SSU, and ordering it to apply a calculated voltage to the actuator. In the frame of this $\ddagger$ http://www.uniservices.co.nz/research/uniservices-centres-of-expertise/biomimetics-lab/self-sensing-unit 


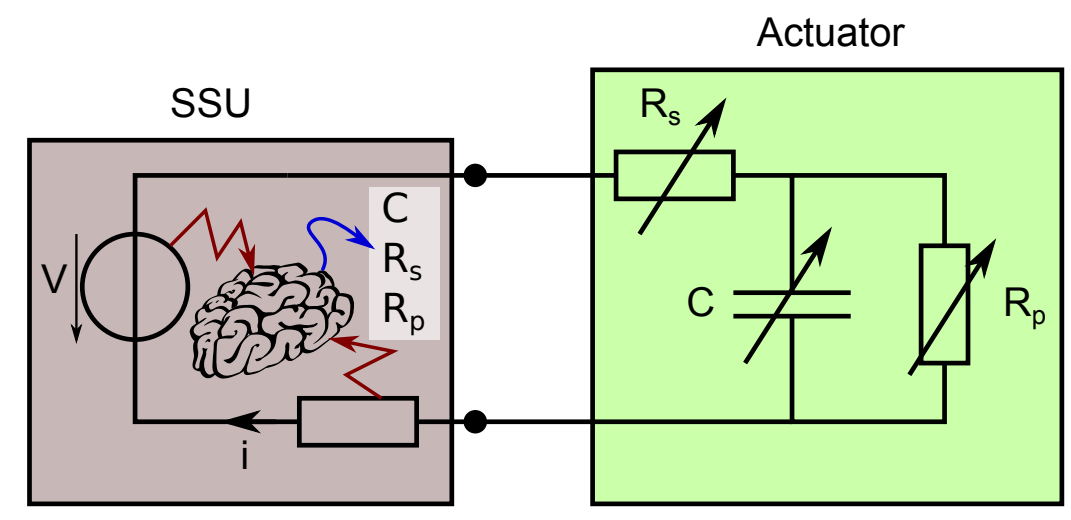

Figure 5. Schematic representation of a DEA driven by the self-sensing unit (SSU). By applying a small time-variant signal superposed to the HV DC signal and measuring the current $i$, the SSU can evaluate the value of the device capacitance $C$, leakage resistance $R_{p}$ and electrode resistance $R_{s}$.

work, in order to demonstrate the application of capacitive self-sensing for closed-loop operation, a simple PI controller was implemented. DEAs have a non-linear response, which is due to the quadratic relationship between the applied field and Maxwell pressure and to the hyperelastic characteristic of elastomers. Consequently, although suited for the purpose of this study, this basic controller configuration is not robust and not suitable for real-world applications. We have shown a method to implement a variable-gain P and PI controller to compensate for the nonlinearities [28]. This method relies on a model of the actuator and allows obtaining dynamic characteristics (steady-state error and response time) which are independent of the set point. Different active control strategies accounting for the DEAs' non linearities have been presented by other research groups $[20,22,29]$. In this work, whose aim is to demonstrate the use of capacitive self-sensing for closed-loop operation, we keep the simple PI approach, but a more robust controller could be implemented with the same hardware.

\section{Fabrication of the tunable grating}

\subsection{Actuators}

The actuators were made with a 3M VHB 4905 membrane (initial thickness $500 \mu \mathrm{m}$ ) prestretched by a factor 3 in both $x$ and $y$ direction for a final thickness of $55.6 \mu \mathrm{m}$ and placed on a PMMA frame. Because VHB is an adhesive, it sticks to the frame, which allows it to keep its prestretch without the need of gluing or mechanical clamping. The electrodes were made with the carbon grease Nyogel 756G and manually applied with a colour shaper. The grating was replicated from a master into a silicone and applied at the center of the membrane (see section 2.2 for more details about the grating replication).

\subsection{Grating replication}

For the DEA tunable grating (figure 2) to work, it is essential that the grating placed at the center of the membrane is soft and deformable, which is not the case of grating structures that can be 

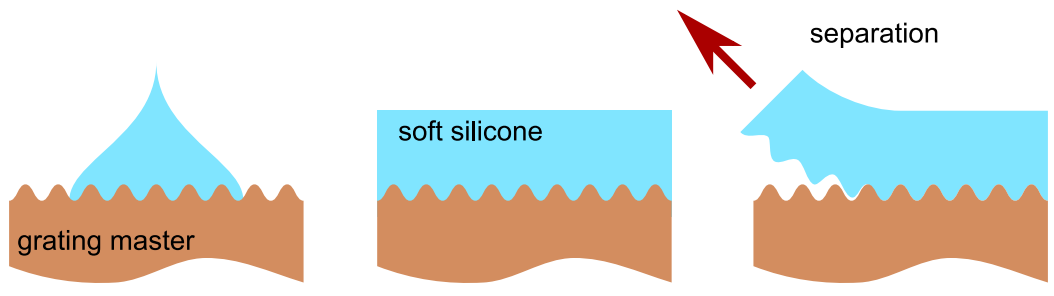

Figure 6. Standard replication process: Liquid silicone is applied on a master grating (left) by casting or spin coating and let to cure. Finally, the silicone with a patterned surface is peeled off from the master (right).
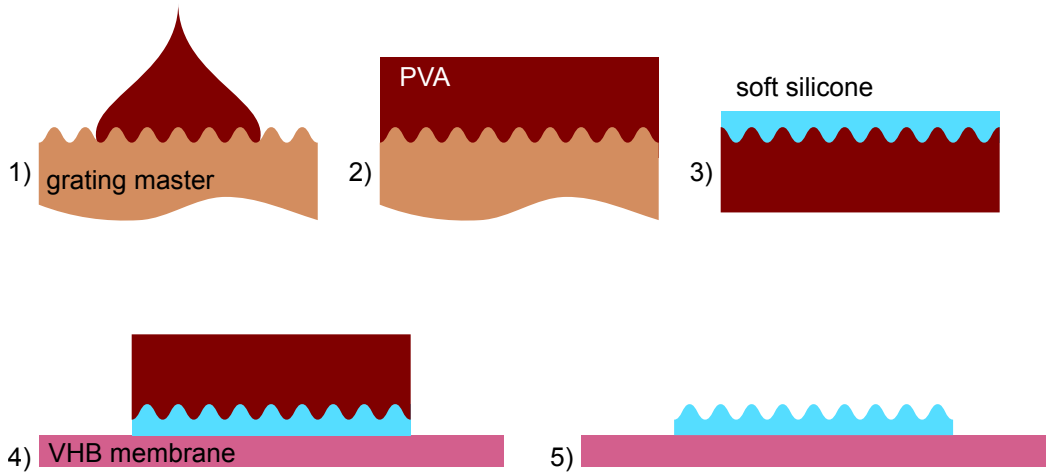

Figure 7. Replication process with a water-soluble master (used in this work): 1) The grating master is placed upside down on a glass plate and the $\mathrm{PVA}: \mathrm{H}_{2} \mathrm{O}$ solution is applied on the grating master. 2) The layer is left to dry. 3) The PVA film is peeled off from the grating sheet, placed upside down on a glass plate and a thin layer of silicone is casted over it. 4) After the silicone has cured, the PVA/silicone sandwich is placed at the center of a prestretched VHB membrane. 5) The PVA master is dissolved under a warm water stream.

commercially bought. Those often consist of a patterned epoxy on a glass substrate (good optical quality, expensive) or a hard plastic sheet that was thermoformed against a master grating (poorer optical quality, cheaper). It is therefore necessary to replicate the grating structure in a material as soft or softer than the dielectric membrane on which the grating is subsequently placed. We used holographic diffraction grating films from Rainbow Symphony as master for the replication with a spatial frequency of 500 lines $/ \mathrm{mm}$.

The classic soft grating replication approach, as reported in the previous works on DEA gratings $[13,14]$, consists in casting a liquid elastomer (typically silicone) on top of a grating master, letting it cure and subsequently peeling the soft grating from the master (figure 6). However, the silicones we used for the grating (Sylgard 186 from Dow Corning and RS 692-542 from RS Components) exhibited a strong adhesion to the master grating sheet, and it was very difficult to separate the silicone layer from master. The need to apply a large force for the separation of the silicone from the master caused breaking of the soft grating when it was made thin. Thicker silicone layers $(>300 \mu \mathrm{m})$ could be successfully separated from the master, but a thicker grating requires more force from the actuator to be deformed, and the tuning range of a DEA tunable grating with a thick grating is considerably reduced. Furthermore, manipulating a soft thin grating is not an easy task, and it is not trivial to place it at the center of the actuator without deforming it and causing optical distortions. Consequently, an alternative replication method was developed. 
The modified process (figure 7) is based on the use of a water-soluble grating master made of a Poly(vinyl alcohol) (PVA). The process involves an additional replication step in which the initial hard plastic master grating is placed upside-down on a glass plate (1), and a layer of PVA is casted on top of it (2). The PVA (Mowiol 4-88 from Aldrich) was first dissolved in warm water with a weight ratio $P V A: \mathrm{H}_{2} \mathrm{O}$ of $1: 2$, leading to a quite thick solution, which can be easily applied in thick layers with a blade applicator (wet thickness: $\sim 200 \mu \mathrm{m}$ ). The layer is left to dry in an oven at $65^{\circ} \mathrm{C}$ and can then be easily peeled off from the grating master. Unlike the silicone layer, which needs a lot of force (and hence strain) to be separated from the hard plastic master, the PVA can be removed quite easily. The obtained layer is flexible, but quite stiff and not stretchable, which prevents straining the grating structure, while making it easy to manipulate. The replicated grating in PVA now serves as a water-soluble master and is placed on a glass plate with the structure facing upwards. A thin layer of soft silicone is casted over the master (3). Because the silicone layer does not need to be separated from the master by pulling on it at a later stage, it can be made much thinner compared to the standard replication process shown on figure 6 , which reduces its stiffness. Once the silicone has cured, the PVA/silicone sheet is cut into $10 \times 10 \mathrm{~mm}^{2}$ squares with a laser cutter. One PVA/silicone sandwich is precisely positioned at the center of a prestretched VHB membrane (prestretch ratio $\lambda_{p s}=3$ in both $x$ and $y$ direction), the silicone side of the sandwich in contact with the adhesive VHB (4). Finally, a flow of lukewarm water is used to dissolve the PVA master and expose the grating. This method presents the advantage of not straining the grating before placing it on the VHB membrane, hence ensuring an optimal grating homogeneity. Very thin gratings can be applied on the VHB membrane with this method. For the current devices, the grating layer was made with blade casting and had a final thickness $>25 \mu \mathrm{m}$, but step 3 could be made by spin-coating, which would allow the manufacturing of gratings in the $2-5 \mu \mathrm{m}$ range. The profile of the Rainbow Symphony master grating and replicated PDMS is shown on figure 8. The Fourier transform of the two profiles gives a spatial frequency of $0.501 \mu \mathrm{m}^{-1}$ for the master and $0.511 \mu \mathrm{m}^{-1}$ for the PDMS grating, separated by the $0.01 \mu \mathrm{m}^{-1}$ resolution of the measurement.

\section{Results}

\subsection{Tunable grating in open and closed loop mode}

To test the actuation scheme based on capacitive self-sensing shown on figure $3 \mathrm{~b}$, a tunable grating actuator was made by positioning a soft silicone grating (500 lines $/ \mathrm{mm}$ ) at the center of a prestretched VHB membrane as described above (prestretch ratio $\lambda_{p s}=3$ in both $x$ and $y$ direction). Then carbon grease electrodes (Nyogel 756G) were manually smeared on the membrane with a color shaper. Two electrode pairs are applied, parallel to the lines patterned on the grating in order to obtain the layout shown on figure 2. The grating being transparent, it is used in transmission mode: a laser (laser diode, $\lambda=670 \mathrm{~nm}$ ) is shone through the grating, the position of the first order diffraction spot is tracked with a camera, and the first order diffraction angle is calculated. The period and grating stretch can then be calculated using the first two lines of equation 5 .

The actuator was first tested in open loop, to check its functionality and evaluate the tuning 


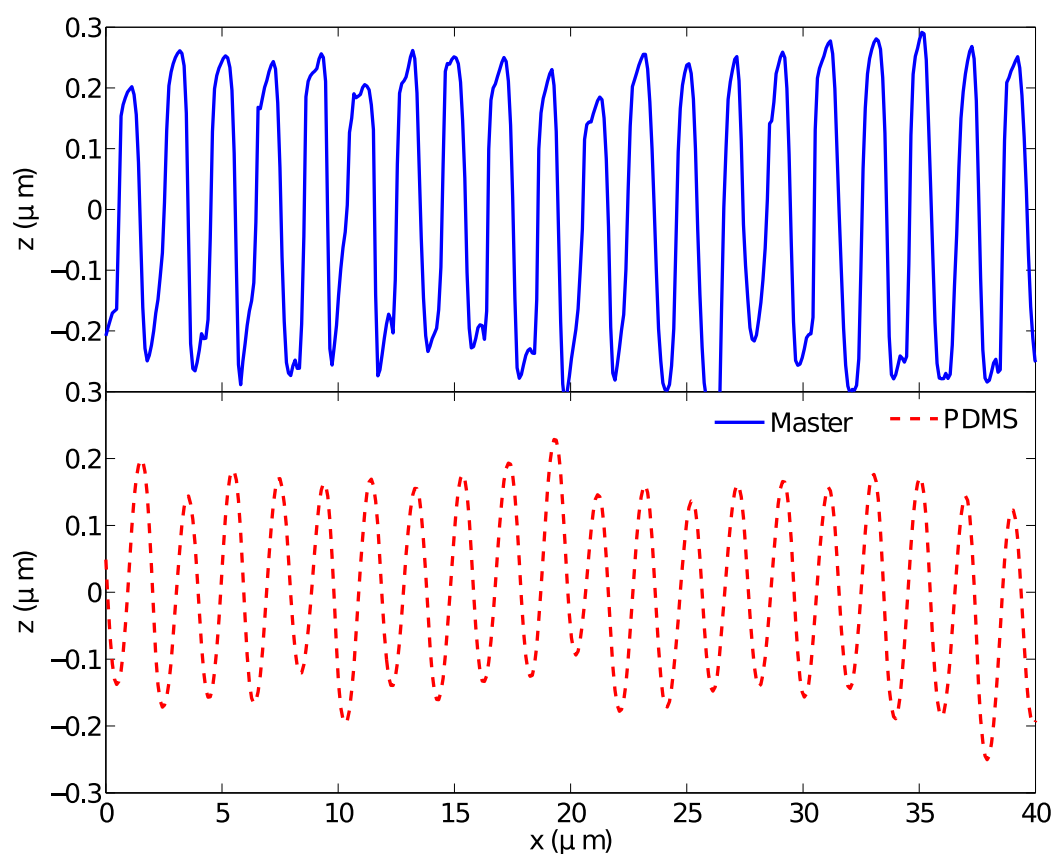

Figure 8. Vertical profile of the master grating sheet (top) and replicated soft PDMS (bottom) measured by white light interferometry. The measured zone on the grating and the master are different.

range of the device. A voltage varying between 0 and $3700 \mathrm{~V}$ was applied to the device. The initial first order diffraction angle is $19.3^{\circ}$ at $0 \mathrm{~V}$, and then steadily increases to $27.1^{\circ}$ at $3700 \mathrm{~V}$, which corresponds to an initial grating period of $2.03 \mu \mathrm{m}$ decreasing to $1.47 \mu \mathrm{m}$ at the maximum tested voltage. The maximal grating stretch is thus 0.73 (or a compressive engineering strain of $-27 \%)$. This is higher than what can be obtained with standard MEMS silicon-based approaches (see e.g. [30,31]). It is is also higher than other published results on DEA-based tunable gratings due to the use of the original replication process based on a water-soluble master. This allows making very thin gratings which do not significantly stiffen the membrane. As a comparison, with a similar DEA tunable grating approach but with a standard grating transfer, Kollosche et al. obtained a maximal compressive strain smaller than 11\% [15]. Because of the standard transfer method used, and the consequent need to peel off the replicated grating from the master and to position it at the center of the actuator, the grating must be stiff enough to be manipulated, and they consequently used a thick film $(75 \mu \mathrm{m})$ of a stiff silicone (DowCorning Sylgard 184), hence explaining the smaller observed strain.

The same actuator was then submitted to a step input both in open loop and closed loop mode (figure 9). In open loop mode, a voltage step of $2700 \mathrm{~V}$ was applied to the device, and its capacitance and grating period measured, respectively with the SSU and the laser diode/camera setup. Due to the viscoelasticity of VHB, the device reacts slowly to the voltage input, which can be seen by the slow response of the capacitance and the grating period change. For the closed-loop operation, a simple PI controller was implemented, using the capacitance given by the SSU as the process variable to regulate. The gain of the proportional part was set to $K_{p}=5$, and the gain of the integral part to $K_{i}=12$. The capacitance set point was set to $1540 \mathrm{pF}$ and was then stepped to $1760 \mathrm{pF}$, a value close to the one reached in open loop mode after $60 \mathrm{~s}$. The capacitance is 

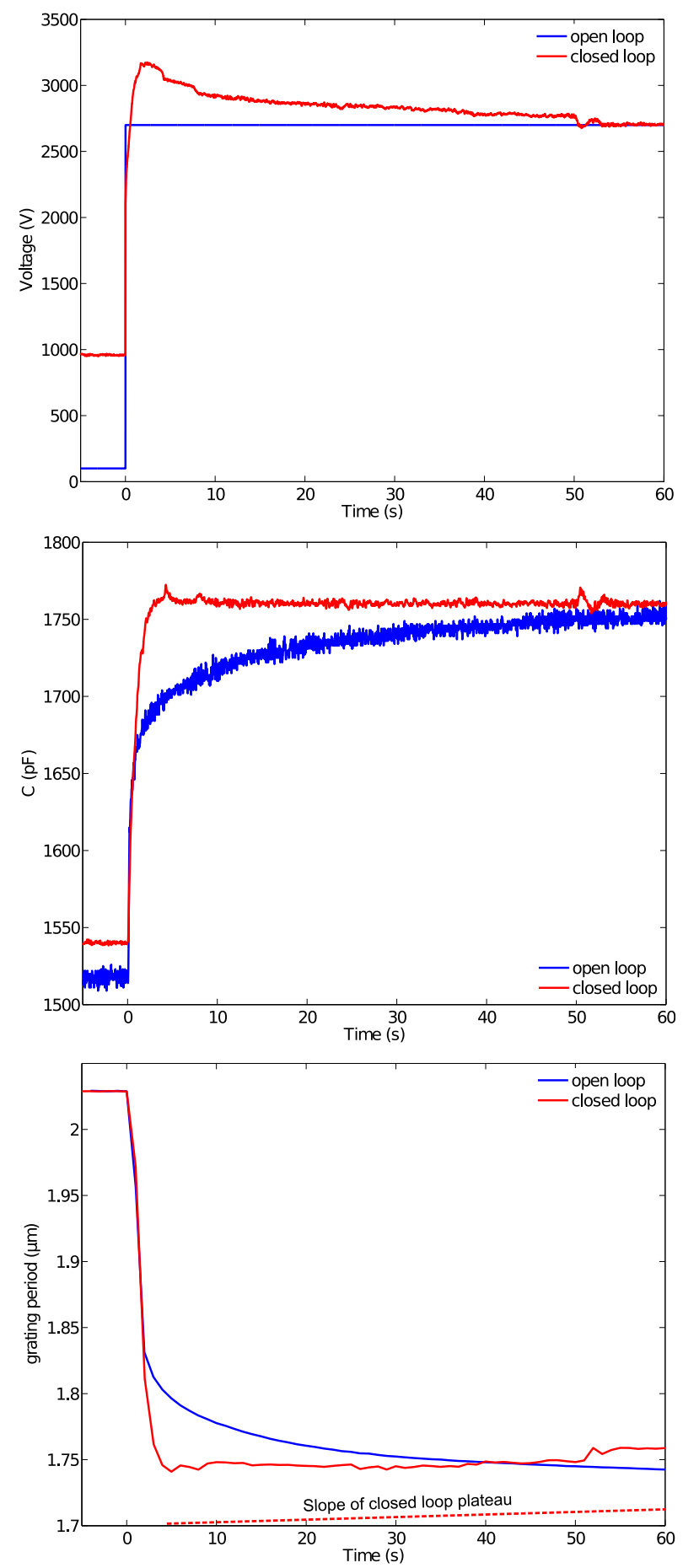

Figure 9. VHB tunable grating in open and closed loop. Top: voltage applied to the actuator. Center: capacitance of the actuator. Bottom: period of the grating (calculated from the measured angle). 
well regulated by the labview interface and SSU (figure 9 center), and it can be seen that the closed-loop actuator is much faster than when driven in open loop. To maintain the constant capacitance value, the controller decreases the voltage over time (figure 9 top). Finally the change in grating period (figure 9 bottom) is much faster in closed-loop mode and varies much less with time. However, some problems and limitations can be observed on the graphs:

- The noise in the capacitance reading causes noise in the calculated value of the applied voltage, and hence noise in the strain of the actuator. This is not clearly visible on the grating period graph, because the camera taking the images is operating at only $10 \mathrm{~Hz}$ vs. $100 \mathrm{~Hz}$ for the voltage and capacitance readings. Noise can be reduced by filtering the capacitance readings or decreasing the gain of the controller, but at the cost of a much slower response. A more complex controller than a standard PI, more adapted to the non-linear relationship between capacitance and voltage, would also help obtaining a more stable output without decreasing the response speed too much. Noise is very detrimental for precision applications such as the tunable grating.

- Although the capacitance is well regulated by the controller and stays at its setpoint value of $1760 \mathrm{pF}$, the grating period slightly drifts and tends to decrease with time. The grating period is expected to keep at a constant value if the capacitance is stable (equation 5), but it is increasing with time, as indicated by the slope of the curve on figure 9 bottom. This needs to be studied in more detail, because if proven, it means that the assumption of a direct link between capacitance and grating period (or diffraction angle) is wrong, at least for a VHB membane. This behaviour would render the use of capacitive self-sensing for closed loop operation of DEAs difficult, especially if the aim is to keep a stable position for an extended period of time. The next section investigates this drifting behaviour in more detail, and shows that it is dependent on the material used as a dielectric membrane. While it is present on VHB-based actuators, those made with silicone are free of it.

Several VHB devices were assembled and characterized, and they all exhibited the same drifting behaviour.

\subsection{Drift of the capacitance-strain relationship}

To further investigate the drift phenomenon observed on the grating actuators, a simpler device was built. Indeed, in the case of the tunable grating, the relation between the capacitance and the calculation of the grating period base on the laser spot displacement goes through a series of linked phenomena, one of which could be the cause of the unforeseen behaviour.

Consequently, we built a simpler device whose base geometry, as shown in figure 10 left, was the same as for the grating actuator, but with a single electrode made from carbon grease. A 3 mm-high rigid piece of laser-cut plastic (PMMA) is placed at the center of the membrane. When the electrode is activated, the rigid bar is shifted on the right, and its displacement is recorded with a precision laser sensor Keyence LC-2400 with a $0.5 \mu \mathrm{m}$ resolution. A 15 minutes closed-loop test was performed, with a capacitance set-point of $890 \mathrm{pF}$ (figure 10 right): the controller can regulate the capacitance to the desired value by controlling the applied voltage, but the displacement drifts during the first 400s of the test. The displacement peaks at $1.62 \mathrm{~mm}$ after $14 \mathrm{~s}$, and then steadily 

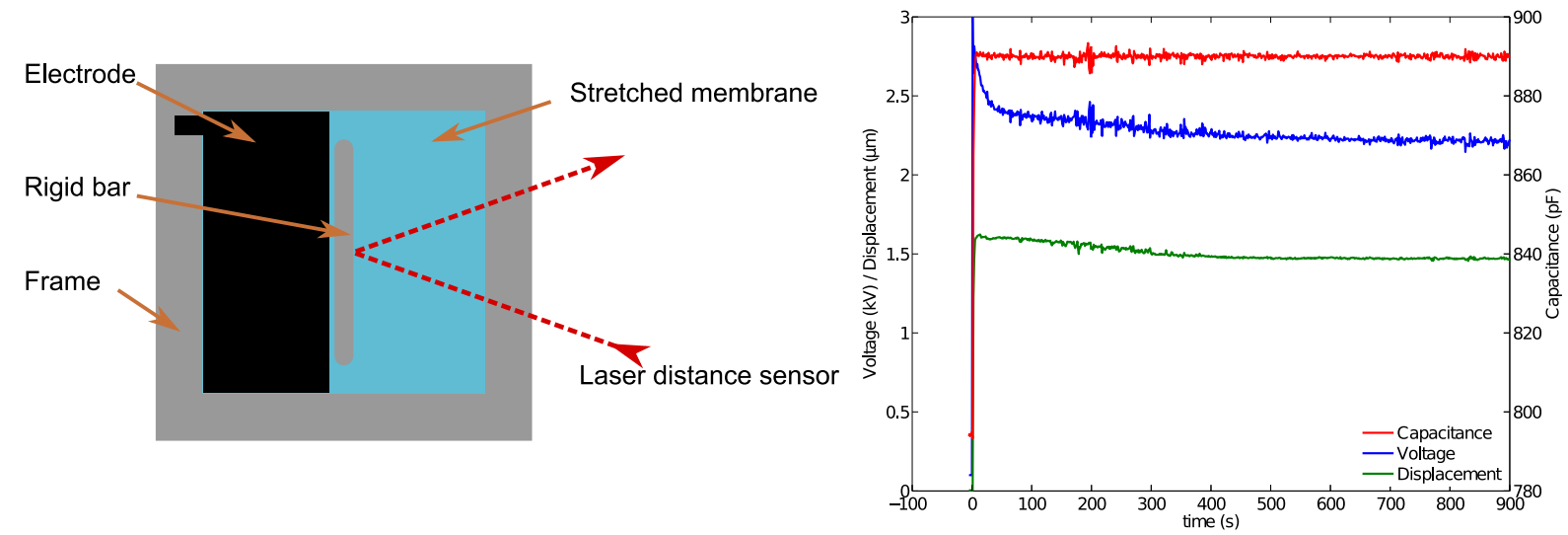

Figure 10. Left: test device consisting of a stretched membrane, a pair of electrode on one side and a rigid part at the center of the membrane. The displacement of the rigid part is tracked with a laser displacement sensor. Right: actuator in closed loop mode. Although the capacitance is kept at a constant value, the displacement drifts during the first $400 \mathrm{~s}$, before stabilizing.
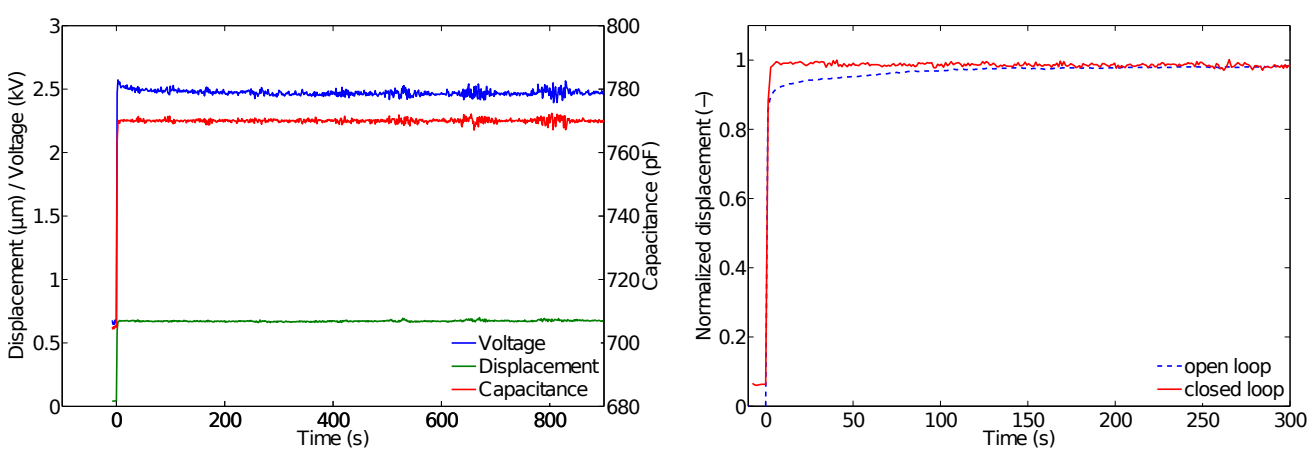

Figure 11. Left: Closed loop operation of the actuator with a silicone membrane. Both the capacitance and the displacement remain constant throughout the test, and no drift is observed. Right: comparison between the displacement in open and closed loop. Closed-loop operation allows for a faster response, but adds noise.

decreases to $1.48 \mathrm{~mm}(-8.65 \%)$ after 400s. The behaviour is similar to what happens with the grating: a relaxation of the strain with time, even though the capacitance of the device doesn't change. To eliminate a possible influence of diffusion of the grease electrodes, a similar test was performed with conductive rubber electrodes (a mixture of silicone and carbon black), which is crosslinked after application and is therefore much more stable than the grease. The actuators with conductive rubber electrodes presented the same drifting behaviour of their capacitance strain relationship, which can therefore not be attributed to grease diffusion or migration.

The drifting behaviour can also be caused by the VHB elastomer, whose viscoelasticity might lead to a time-dependent relative permittivity $\epsilon_{r}$, either due to stress relaxation in the material, or by a slow orientation of polar parts of the elastomer chains under the effect of the high electric field. The relative permittivity of VHB is known to have an unexpected behaviour and to depend on prestretch and applied field [32]. The results of this study tend to show that there is also a time-dependence of the permittivity when the material is submitted to a high electric field. The observed drift is not due to stress relaxation or creep induced by the prestretch of the membrane, because the phenomenon is repeatable and subsequent tests on the same device show the same 
behavior. It should also be pointed out that prestretch-induced stress relaxation in the viscoelastic membrane causes a change of the strain-voltage characteristic because of the non-linear mechanical properties of the VHB membrane. However, the self-sensing closed-loop approach is insensitive to this effect, because the relation between capacitance and strain remains unaffected. But to avoid any undesired influence of stress relaxation on the experiments, a period of 24 hours has systematically been observed between the prestretching of the membrane and the testing of the device. Experimentally, a slight decrease of the capacitance with time has been observed on the samples with carbon grease electrodes. This is effect is probably due to diffusion of the oily component from the grease into the membrane, which increases its thickness and/or influences its relative permittivity. The observed drift is about $5 \mathrm{pF}$ per day and does not influence the results presented here, as the acquisition time used was 15 minutes or less.

Because other dielectric elastomer materials might not present the same behaviour, we tested an actuator made with a silicone elastomeric membrane, which was prepared by casting the one component silicone RS 692-542 (RS Components) on a glass plate previously coated with a watersoluble sacrificial layer. Once the silicone has crosslinked, the membrane is removed from the glass plate and prestretched equi-bixially by a factor 1.55 before being glued on a frame. Nyogel $756 \mathrm{G}$ grease electrodes are then applied on the membrane. Except for the dielectric material, the actuator is identical to the one used for the tests on VHB (figure 10 left). Similar to what was done with VHB, the actuator is driven in closed loop-mode for 15 minutes with an initial set point jump at $t=0$ from $C_{0}$ to $770 \mathrm{pF}$ (figure 11 left). Unlike what was observed for VHB, and although the applied electric field is equivalent, no drift between the capacitance and the displacement is observed. The displacement, however is about one third the value for VHB, thus showing why the latter material is often preferred by researchers despite its pitfalls. Figure 11 right compares the displacement obtained with the silicone actuator in open and closed-loop modes. Closed loop operation allows for a faster response speed: it takes about $3 \mathrm{~s}$ for the actuator to reach $90 \%$ of its final displacement in open loop, whereas in closed-loop mode, the rise time is 3 times shorter, and the displacement remains at the final value during the complete test duration (15 min), and doesn't present a time dependent drift as it was the case for VHB. Noise in the capacitance measurement system leads to noise in the computation of the error (difference between capacitance set point and actual value), which in turns leads to noise in the applied voltage and hence in the measured displacement, as can be seen on figure 11. Because of the quadratic relation between the strain and applied voltage, the noise in the actuator strain depends on the set point and increases for higher capacitive set points (i.e. higher applied voltage and higher output strain). This is one of the limitation of the standard PI controller when driving non-linear systems, which can be solved by using more advanced controlling algorithms.

\section{Conclusion}

Compared to the traditional approach of negative feedback, which requires an external sensor, capacitive self sensing is an elegant approach to drive DEAs in closed loop because it does not require any additional sensing element. And because of its slow response speed and viscoelastic drift and creep, VHB acrylic elastomer-based DEAs would particularly benefit from closed loop mode operation, which can both increase the actuator speed and stabilize its output. However, 
because of the drift between the device strain and the capacitance of its electrode, this approach is not suitable for VHB dielectric membranes if displacement control better than $1 \%$ is needed. It can still be used to increase the response speed of the device, but a longer-term stable position cannot be held, probably because of a time-dependent $\epsilon_{r}$, linked to either stress relaxation or molecules orientation. A tunable grating made with this membrane material and a thin deformable silicone grating replicated on a water-soluble master exhibited a very large tuning range of $27 \%$ of the grating period. However, it was not able to hold a precise position, even when operated with capacitive feedback, due to the behaviour of the VHB dielectric membrane.

On the other hand, silicone membranes didn't present a drift between their strain and capacitance, and can therefore be used in combination with capacitive self-sensing for fast and precise actuation. Noise in the capacitance reading can be problematic, because it leads to noise in the actuation voltage and output strain. This issue can be solved by an optimization of the selfsensing unit and associated algorithm to compute the capacitance, as well as by an optimization of the feedback controller. A standard PI controller is not the best choice to drive a DEA, because of the non-linear relationship between voltage and capacitance, rendering the device much more sensitive to capacitance reading noise at the high driving voltage used for the tests presented here. However, by combining the best materials (silicones) with a carefully designed controller, the use of capacitive self-sensing would open a wider field of application to DEAs by adding precise and stable displacement to the already outstanding strain.

\section{Acknowledgments}

The authors wish to thank the Swiss National Science Foundation (grant IZK0Z2-141692), which funded the visit of S. Rosset to the Biomimetics lab and Mr. E. Wax for fruitful discussions.

\section{References}

[1] Tiefeng Li, Christoph Keplinger, Richard Baumgartner, Siegfried Bauer, Wei Yang, and Zhigang Suo. Giant voltage-induced deformation in dielectric elastomers near the verge of snap-through instability. Journal of the Mechanics and Physics of Solids, 61(2):611 - 628, 2013.

[2] R. E. Pelrine, R. D. Kornbluh, and J. P. Joseph. Electrostriction of polymer dielectrics with compliant electrodes as a means of actuation. Sensors and Actuators, A: Physical, 64(1):77-85, 1998.

[3] Samuel Rosset and HerbertR. Shea. Flexible and stretchable electrodes for dielectric elastomer actuators. Applied Physics A, 110:281-307, 2013.

[4] Philippe Jean, Ambroise Wattez, Guillaume Ardoise, C. Melis, R. Van Kessel, A. Fourmon, E. Barrabino, J. Heemskerk, and J. P. Queau. Standing wave tube electro active polymer wave energy converter. In Yoseph Bar-Cohen, editor, Proceedings of SPIE - The International Society for Optical Engineering, volume 8340, page 83400C. SPIE, 2012.

[5] Thomas G. McKay, Benjamin M. O'Brien, Emilio P. Calius, and Iain A. Anderson. Soft generators using dielectric elastomers. Applied Physics Letters, 98(14):142903, 2011.

[6] Roy D. Kornbluh, Ron Pelrine, Harsha Prahlad, Annjoe Wong-Foy, Brian McCoy, Susan Kim, Joseph Eckerle, and Tom Low. From boots to buoys: promises and challenges of dielectric elastomer energy harvesting. In Yoseph Bar-Cohen and Federico Carpi, editors, Proceedings of SPIE - The International Society for Optical Engineering, volume 7976, page 797605. SPIE, 2011.

[7] Samin Akbari and Herbert R. Shea. An array of 100um x 100um dielectric elastomer actuators with $80 \%$ strain for tissue engineering applications. Sensors and Actuators A: Physical, 186:236 - 241, 2012. 
[8] Marc Matysek, Peter Lotz, Klaus Flittner, and Helmut F. Schlaak. Vibrotactile display for mobile applications based on dielectric elastomer stack actuators. In Yoseph Bar-Cohen, editor, Proceedings of SPIE - The International Society for Optical Engineering, volume 7642, page 76420D. SPIE, 2010.

[9] M. Matysek, P. Lotz, T. Winterstein, and H.F. Schlaak. Dielectric elastomer actuators for tactile displays. In Proceedings - 3rd Joint EuroHaptics Conference and Symposium on Haptic Interfaces for Virtual Environment and Teleoperator Systems, World Haptics 2009, pages 290-295, 2009.

[10] I.A. Anderson, T. Hale, T. Gisby, T. Inamura, T. McKay, B. O'Brien, S. Walbran, and E.P. Calius. A thin membrane artificial muscle rotary motor. Applied Physics A: Materials Science and Processing, 98(1):75-83, 2010.

[11] Iain A. Anderson, Todd A. Gisby, Thomas G. McKay, Benjamin M. O'Brien, and Emilio P. Calius. Multifunctional dielectric elastomer artificial muscles for soft and smart machines. Journal of Applied Physics, 112(4):041101, 2012.

[12] Federico Carpi, Gabriele Frediani, Simona Turco, and Danilo De Rossi. Bioinspired tunable lens with musclelike electroactive elastomers. Advanced Functional Materials, 21(21):4152-4158, 2011.

[13] Manuel Aschwanden and Andreas Stemmer. Low voltage, highly tunable diffraction grating based on dielectric elastomer actuators. In Proceedings of SPIE - The International Society for Optical Engineering, volume 6524, pages 65241N-10, San Diego, California, USA, 2007. SPIE.

[14] Manuel Aschwanden, David Niederer, and Andreas Stemmer. Tunable transmission gratings based on dielectric elastomer actuators. In Electroactive Polymer Actuators and Devices (EAPAD) 2008, volume 6927, pages 6927-56, San Diego, California, USA, 2008. SPIE.

[15] Matthias Kollosche, Sebastian Doering, Joachim Stumpe, and Guggi Kofod. Voltage-controlled compression for period tuning of optical surface relief gratings. Opt. Lett., 36(8):1389-1391, Apr 2011.

[16] Markus Beck, Reto Fiolka, and Andreas Stemmer. Variable phase retarder made of a dielectric elastomer actuator. Opt. Lett., 34(6):803-805, Mar 2009.

[17] Ron Pelrine, Roy Kornbluh, Jose Joseph, Richard Heydt, Qibing Pei, and Seiki Chiba. High-field deformation of elastomeric dielectrics for actuators. Materials Science and Engineering C, 11(2):89-100, 2000.

[18] P. Brochu and Q. Pei. Advances in dielectric elastomers for actuators and artificial muscles. Macromolecular Rapid Communications, 31(1):10-36, 2010.

[19] R. Sarban, J. Oubaek, and R.W. Jones. Closed-loop control of a core free rolled eap actuator. In Proceedings of SPIE - The International Society for Optical Engineering, volume 7287, page 72870G, 2009.

[20] Rahimullah Sarban and Richard W. Jones. Physical model-based internal model control of a de actuator. In Proceedings of SPIE - The International Society for Optical Engineering, volume 7976, pages 79761J79761J-14, 2011.

[21] Marco Randazzo, Matteo Fumagalli, Giorgio Metta, and Giulio Sandini. Closed loop control of a rotational joint driven by two antagonistic dielectric elastomer actuators. In Proceedings of SPIE - The International Society for Optical Engineering, volume 7642, pages 76422D-76422D-12, 2010.

[22] W. Kaal and S. Herold. Electroactive polymer actuators in dynamic applications. Mechatronics, IEEE/ASME Transactions on, 16(1):24-32, 2011.

[23] Benjamin O'Brien, Justin Thode, Iain Anderson, Emilio Calius, Enrico Haemmerle, and Shane Xie. Integrated extension sensor based on resistance and voltage measurement for a dielectric elastomer. In Proceedings of SPIE - The International Society for Optical Engineering, volume 6524, pages 652415-11. SPIE, 2007.

[24] T.A. Gisby, S. Xie, E.P. Calius, and I.A. Anderson. Integrated sensing and actuation of muscle-like actuators. In Proceedings of SPIE - The International Society for Optical Engineering, volume 7287, pages 728707-1, 2009.

[25] Todd A. Gisby, Benjamin M. O'Brien, Sheng Q. Xie, Emilio P. Calius, and Iain A. Anderson. Closed loop control of dielectric elastomer actuators. In Proceedings of SPIE - The International Society for Optical Engineering, volume 7976, pages 797620-797620-9, 2011.

[26] Todd A. Gisby, Benjamin M. O'Brien, and Iain A. Anderson. How far and how hard: tactile feedback for robotic manipulators. In Yoseph Bar-Cohen, editor, Proceedings of SPIE - The International Society for Optical Engineering, volume 8340, page 83401S. SPIE, 2012.

[27] Todd A. Gisby, Benjamin M. O'Brien, and Iain A. Anderson. Self sensing feedback for dielectric elastomer actuators. submitted to Applied Physics Letters. 
[28] S. Rosset, B. O'Brien, T. Gisby, D. Xu, H. Shea, and I. Anderson. Tunable grating with active feedback. In Proceedings of SPIE - The International Society for Optical Engineering, volume 8687, page 868768, 2013.

[29] J. Maas, C. Graf, and L. Eitzen. Control concepts for dielectric elastomer actuators. In Proceedings of SPIE - The International Society for Optical Engineering, volume 7976, page 79761H, 2011.

[30] Maurizio Tormen, Yves-Alain Peter, Philippe Niedermann, Arno Hoogerwerf, and Ross Stanley. Deformable mems grating for wide tunability and high operating speed. Journal of Optics A: Pure and Applied Optics, 8(7):S337, 2006.

[31] Chee Wei Wong, Yongbae Jeon, George Barbastathis, and Sang-Gook Kim. Analog tunable gratings driven by thin-film piezoelectric microelectromechanical actuators. Appl. Opt., 42(4):621-626, Feb 2003.

[32] T.G. Mckay, E. Calius, and I.A. Anderson. The dielectric constant of $3 \mathrm{~m}$ vhb: A parameter in dispute. In Proceedings of SPIE - The International Society for Optical Engineering, volume 7287, page 72870P, 2009. 